\title{
PROPUESTA METODOLÓGICA PARA ANALIZAR FENÓMENOS SOCIO TÉCNICOS EN INTERNET
}

\section{METHODOLOGICAL PROPOSAL TO ANALYZE SOCIOTECHNICAL PHENOMENA ON THE INTERNET}

\author{
PROPOSTA METODOLÓGICA PARA ANALISAR FENÔMENOS \\ SOCIOTEQUÍNICOS NA INTERNET
}

\author{
Ramón Abraham Mena Farrera ${ }^{1}$
}

\begin{abstract}
Resumen: No podemos soslayar que las experiencias humanas están vinculadas a la vigorosa expansión tecnológica de los medios electrónicos de comunicación. Por tanto, el presente artículo presenta una revisión teórica que permite formular una estrategia en la metodología cualitativa para producir, ordenar y analizar nuevos datos empíricos que nos den cuenta del fenómeno de la interacción humana en la red de internet. La estrategia metodológica propuesta se sostiene en las tradiciones filosóficas y sociológicas de la construcción social de la tecnología (WINNER, 1993; BIJKER, 1987; PINCH; BIJKER, 1987), la teoría del actor-red (LAW, 1992; CALLON, 1998), y el modelo de los sistemas (HUGHES, 1983), usados para la comprensión de los sistemas socio tecnológicos. Se concluye con algunos posicionamientos renovadores para la metodología cualitativa y su presencia para analizar fenómenos de internet inspirados en la fenomenología, enfatizando el rol concreto del individuo en sus experiencias (mundos vividos y significativos) y el hecho de la acción intencional que tiene el interlocutor de internet para compartir sus vivencias.
\end{abstract}

Pablas clave: Relaciones socio técnicas; Fenomenología tecnológica; Etnometodología; Internet.

\begin{abstract}
We cannot ignore that human experiences are linked to the vigorous technological expansion of electronic media. Therefore, this article presents a theoretical review that allows us to formulate a strategy in the qualitative methodology to produce, organize and analyze new empirical data that give us an account of the phenomenon of human interaction in the internet network. The proposed methodological strategy is based on the philosophical and sociological traditions of the social construction of technology (WINNER, 1993; BIJKER, 1987; PINCH; BIJKER, 1987), the actor-network theory (LAW, 1992; CALLON, 1998), and the systems model (HUGHES, 1983), used for the understanding of sociotechnological systems. It concludes with some innovative positions for the qualitative methodology and its presence to analyze internet phenomena inspired by phenomenology, emphasizing the concrete role of the individual in their experiences (lived and significant worlds) and the fact of the intentional action of the interlocutor of Internet to share your experiences.
\end{abstract}

Keywords: Sociotechnical relations; Technological phenomenology; Ethnomethodology; Internet.

Resumo: Não podemos ignorar que as experiencias humanas estão vinculadas à vigorosa expansão tecnológica dos meios eletrônicos de comunicação. Assim, o presente artigo apresenta uma revisão teórica que permite formular uma estratégia metodológica qualitativa para produzir, ordenar e analizar novos dados empíricos que permitam dar conta do fenômeno da interação humana na rede de internet. A estratégia metodológica proposta se sustenta nas tradições filosóficas e sociológicas da construção social da

${ }^{1}$ Doctor en Ciencias Sociales y Humanidades por El Centro de Estudios Superiores de México y Centroamérica (CESMECA) de la Universidad de Ciencias y Artes de Chiapas (México) y La Universidad de Alicante (España). Académico del Departamento de Sociedad y Cultura de El Colegio de la Frontera Sur, México. E-mail: rmena@ecosur.mx 
tecnologia (WINNER, 1993; BIJKER, 1987; PINCH; BIJKER, 1987), na teoria ator-rede (LAW, 1992; CALLON, 1998) e no modelo dos sistemas (HUGHES, 1983) usado para a compreensão dos sistemas sócio-tecnológicos. Conclui-se o texto com uma releitura de posicionamentos sobre a metodologia de pesquisa qualitativa, bem como a sua presença para analisar fenômenos da Internet inspirados na fenomenologia, enfatizando o conjunto de experiências vividas de cada indivíduo (mundos e estados vividos), bem como o fato de admitir as ações intencionais do interlocutor com a Internet e o modo como compartilham suas informações e experiências.

Palavras-chave: Relações sociotécnicas; Fenomenologia tecnológica; Etnometodologia; Internet.

\section{Introducción}

Para comprender el fenómeno de la experiencia en la red de internet se requieren analizar los relatos que los interlocutores construyen en la red, ya sea como imagen o discurso narrativo dentro de múltiples sitios digitales. Para lograrlo se necesita identificar un corpus de discursos y prácticas más o menos homogéneas que dentro de las relaciones socio técnicas sean buscados, seleccionado y consumidos por los propios usuarios de la red. En los espacios digitales se producen contactos directos, complejos y virtuales con otras personas dentro de la red, estableciendo coplejas relaciones en la dimensión sociotécnica entre los interlocutores El contacto, las alteridades e identificaciones permitirán al interlocutor entrar en diversos procesos de reflexividad social.

Dicho de otra manera, una experiencia en la red de internet puede proyectarse, y situarnos en múltiples experiencias sociotécnicas apelando a las claves que crean interacciones más allá de los muros físicos de nuestro mundo material y permitirnos teorizar y cuestionarnos acerca de una o muchas experiencias vividas en la red. De lo que se trata, es de definir claramente los ámbitos de análisis en donde se expresan y sobreponen las interacciones y experiencias tecnológicas, comerciales, políticas y humanas.

Si deseamos comprender la experiencia humana contemporánea, la red de internet ofrece espacios que pertenecen al mundo cotidiano de personas de distintos barrios, ciudades, países o continentes las cuales comparten y se interconectan unas a otras a través de esos espacios siempre actualizados, diseñados, mantenidos y que expresan de forma digital la vida cotidiana. Es más, los mismos paisajes representan una importante fuente de información, destacando a partir de su estructura discursiva y por la escritura situacional que se registran en los textos de chats, blogs o redes sociales. La práctica discursiva envuelve a los interlocutores en una aparente y fantasmagórica representación sobre el consumo, las relaciones de poder, la fantasía, el placer, la libertad, la compañía 
que se da en la sensación del encuentro con el otro, o en la aventura del conocimiento de las llamadas "nuevas tecnologías".

Este articulo tiene el propósito de aportar a la tradición metodológica cualitativa elementos que resuelvan el reto de responder cómo situarnos onto epistémicamente para producir, organizar y sistematizar la producción de datos que producen las interacciones virtuales. Lo anterior nos llevan a preguntarnos ¿Cómo podemos crear metodologías cualitativas para la comprensión la experiencia concreta de quienes, en el paisaje tecnológico, interactúa en relación con la tecnología (interlocutor-artefacto técnico), el mercado (interlocutor-consumo), la política (interlocutor-poder) y la dimensión humana (interlocutor-interlocutor)?

Sin duda el reto metodológico que nos presenta la internet se sintetiza en construir estrategias rigurosas que nos permitan conocer, documentar, sistematizar y analizar ¿Cuál es la forma de habitar estos paisajes en tanto representa otros mundos de la vida virtual? ¿Qué lugar ocupa la experiencia en la red dentro de su vida cotidiana?

Lo anterior adquiere importancia global para las ciencias sociales y humanísticas cuando consideramos la escala de conexión en internet. Hablamos de la interacción de millones de personas de todas las edades, orígenes, razas, géneros, y niveles sociotécnicos los cuales nos obliga a plantear nuevos objetivos en los métodos de estudio en las redes, planteando nuevos objetivos como los son: el diseñar la construcción de una serie de cualidades que son reflexionadas para fortalecer la definición de identidad en la red, ya sea como simulacro, la alteridad virtual y el encuentro con referentes identitarios que dan sentido a su vida, las cuales nos permitan resolver la tensión ante una vehemente vulnerabilidad tecnológica (el control estatal, de las empresas, y otros actores de poder), la mercantilización del espacio virtual, nuevas expresiones del poder, la virtualidad del otro y una constante exposición a la seguridad privada burlada.

\section{Interés metodológico para comprender el fenómeno en la red}

Los estudios comprensivos que nos lleva a una aproximación teóricometodológica desde la perspectiva de las ciencias sociales y fenomenología tecnológica nos permiten situarnos en la experiencia como fenómeno, guiándonos en la comprensión de dicha experiencia personal, principalmente, sobre la tecnológica, para luego abordar el consumo, la política y las relaciones humanas en situaciones particulares, en paisajes tecnológicos concretos. Lo anterior permite identificar la experiencia de los interlocutores 
que usan el internet y, en ella, reconocer rasgos particulares de sus interrelaciones humanas, que por la red constituyen disposiciones emocionales diversas (sentimientos, situaciones, emociones, estados de ánimo y afecciones).

Los textos en internet son el elemento primordial para la caracterización del paisaje tecnológico dentro de cualquier trabajo que en la red de internet se quiera realizar un trabajo de campo. Para quienes elegimos a internet como un espacio para realizar el trabajo de campo se debe considerar que un análisis del texto nos debe llevar a la construcción del dato en donde la narrativa, en el texto y el habla, median y conforman eventos socialmente significativos de los interlocutores por medio de internet. Llegar a ello podría llevar a la persona que investigue a la producción de datos a partir del análisis de los relatos textuales, para luego realizar cuestionarios cerrados, vía internet, que permitan la producción de información acerca de los interlocutores y autores de cualquier relato. Para precisar lo anterior, es importante puntualizar que la producción está dada por medio de relatos de vida, mismos pueden ser interpretados a partir de unidades de análisis las cuales estructuran metodológicamente a las relaciones socio técnicas, mediante las cuales se den representaciones individuales sobre la experiencia tecnológica, política, económica y humana y, fundamentalmente, las expresiones concretas del fenómeno de la interacción humana por medio de la tecnología.

Lo anterior se sostiene en el hecho de que entre la experiencia vivida y la narración de la experiencia existe una relación que establece la identidad constitutiva: “...no hay identidad cultural que no sea contada..." (SANTAMARINA; MARINAS, 2007, p. 57). Esta relación entre narración y reconocimiento de la identidad se hace visible a través del lenguaje y el diálogo, que constituye y transforma tanto al narrador como a su interlocutor. La identidad no es lo que se le atribuye a alguien por el hecho de pertenecer a un grupo, sino la "expresión" que da sentido y valor a la vida del individuo. La identidad individual y colectiva depende y vive del reconocimiento de los otros, se construye en la narración y el intercambio intersubjetivo.

La producción del dato en la red puede definirse en cualquier investigación en tres momentos. El primero está representado por los aspectos generales de la metodología de análisis de las experiencias de relaciones humanas en redes socio técnicas basadas en internet, de la cual se desprende un diagrama de categorías conceptuales, que pasan a ser categorías analíticas a priori. En un segundo momento, se hace el abordaje del análisis del material en internet que evidencian las influencias recíprocas entre redes sociales, blogs, chat y cualquier espacio de internet en donde se considera a las estructuras de 
narrativas de internet como tramas de hiperenlaces con las cuales se consideren los aspectos innovadores del análisis de relaciones socio técnicas. En ellas se consolida el uso de las categorías y surgen las categorías de análisis in vivo. Por último, el material se discute específicamente la metodología utilizada para realizar los relatos de vida, los cuales nos llevan a tener un contacto cara a cara con los interlocutores que escriben y alimentan cualquier plataforma de internet, tales como blogs, redes sociales y conversaciones mediante mensajeros electrónicos. Los tres momentos anteriores de producción del dato buscan separar fenómenos cuya percepción y análisis se potencian en la alianza entre abordajes histórico - interpretativos y estructurales, estimulados por los mapas de las redes socio técnicas establecidas en internet.

\section{Propuesta metodológica para analizar los fenómenos en Internet}

Los estudios que se realizan en la red de internet deberán proponer metodológicamente por lo menos cuatro momentos en donde se explicite la tradición de estudios sociales y filosóficos acerca de la tecnología, el posicionamiento sobre los enfoques teóricos frente a la tecnología - determinismo tecnológico, comunidades científicas, impulso tecnológico y la sociedad del conocimiento — los cuales tengan como fin contextualizar el problema de investigación en el marco del compendio histórico entre ciencia y tecnología. Algunos debates acerca de la tecnología como los presentados por Winner (1979), Ellul (1960), Quintanilla (2001), Smith y Marx (1996), Heilbroner (1996), Bunge (1972), Wienner (1993), Wynne, (1983) y Hughes (1983). Cabe aclarar que los debates filosóficos sobre la tecnología se realizan mucho antes de la era de internet, y la reflexión sobre los medios de comunicación, los instrumentos tecnológicos, las interacciones humanas a través de los instrumentos tecnológicos no inician con internet ni terminaran con el fin de su dominio.

A pesar de que las reflexiones filosóficas y la discusión sobre la tecnología no se agota en ella misma, sino que complementan los estudios sociales de la ciencia — ciencia en acción en contra del determinismo, etnometodología de la actividad científica, procesos de reflexividad en la organización social y la teoría de la acción comunicativa, en donde se articulan las perspectivas y su tránsito al abordaje filosófico. Lo anterior permite situarnos en una tradición que estudia los fenómenos socio técnicos ${ }^{2}$ para

\footnotetext{
${ }^{2}$ Bijker (1987) explica que los fenómenos, enfoques y artefactos sociotécnicos no pueden ser considerados en concepto autoexplicativo, no tiene razones internas, inmanentes o intrínsecas que lo expliquen por sí mismo más allá de sus diversas interrelaciones sociales, técnicas, económicas, políticas que lo constituyen
} 
entonces usar las categorías conceptuales que llevan a analizar textos con episodios significativos de la representación tecnológica, y así comparar los significados y las prácticas de los interlocutores, comprendiendo las cualidades emergentes de las interacciones virtuales a partir de la reflexividad de los interlocutores sobre sus experiencias en la red de internet. En la discusión se destacan autores que guiaron nuestra construcción teórica como Merton (1953), Winner (1993 [1979]), Bijker (1987), Garfinkel (2006), Espinosa, Giner y Torres (2002), Kreimer (2004), Thomas (2009) y Habermas (1988).

\section{Enfoques teóricos frente a la tecnología}

Las reflexiones teóricas son históricamente insuficientes para sostener un debate constante y actualizado en el debate sobre la tecnología. Se reconoce que el valor central de los estudios sobre tecnología se da en el desarrollo de la creatividad intelectual, al pensarla como un sistema social complejo, al derrumbar las posiciones del determinismo tecnológico, y en el transitar de una época a otra, consolidándose un pensamiento renovador, en donde los actores sociales logran crear innovaciones sociales y culturales. Nadie niega que en muchos casos la sociedad es deslumbrada por los nuevos modelos y los costos de un sinnúmero artefactos electrónicos. Sin embargo, la intensa oferta está centrada no en el valor del objeto, sino en la posibilidad de conexión con otros seres humanos y las posibilidades que esto puede generar.

El debate del carácter artefactual de la tecnología como fuente de explicación del determinismo tecnológico se refleja en la posición de Heilbroner (1996), quien identifica ciertos momentos históricos que relacionan el determinismo con la tecnología de la producción, a partir de unas secuencias fijas a seguir por la tecnología, las cuales serían consecuencias lógicas de la conquista técnica de la naturaleza. En lo cognitivo Bunge (1972), considerado como uno de los principales expositores de este enfoque cognitivo o intelectualista, considera a la tecnología como "ciencia aplicada y a la propia ciencia como una búsqueda por nuevas leyes de la naturaleza, la cual es conducida por la arrogancia y la libertad del espíritu” (BUNGE, 1972, p. 143).

históricamente. Este enfoque sociotécnico reconstruye analíticamente tanto el momento dónde los artefactos tecnológicos son partes constitutivas de las relaciones sociales, políticas, económicas como al momento en que estas diversas relaciones se materializan en artefactos tecnológicos. Es decir, analiza cómo las tecnologías son construidas socialmente y cómo las sociedades son construidas tecnológicamente. 
Mientras tanto Hughes (1983) propone un modelo para la comprensión de un sistema tecnológico. A partir de su estudio Networks of Power (1983), muestra que los sistemas tecnológicos están constituidos por componentes complejos y heterogéneos. Los componentes de los sistemas tecnológicos pueden ser artefactos físicos (computadoras, redes, dispositivos), organizaciones (tales como empresas de conexión a internet, compañías de servicio público), asuntos usualmente descritos como científicos (libros, artículos, manuales, investigación), artefactos legislativos (tales como leyes) e, igualmente, los recursos naturales pueden ser considerados como artefactos de un sistema tecnológico.

Por tanto lo artefactual, cognitivo y sistémico se sintetiza, y es recreada la mayoría de las veces, cuando alguien cambia o actualiza algún equipo de comunicación electrónica, léase computadora o teléfono celular, por citar un par de ejemplos. Un nuevo objeto representa un nuevo artefacto en lo concreto y, en sí mismo, es una caja tecnológica. El objeto contará, la mayoría de las veces, con una renovada interfaz a la cual hay que acostumbrarse para moverse en ella. El poseedor del artefacto se decide a preguntar y confiar en otros a través de los manuales, las tutorías y la red humana interconectada por medio de otros artefactos similares en una red de telecomunicación. La elección será confiar, por así decirlo, en una intuición tecnológica, que no es otra cosa que la síntesis de la propia experiencia en la relación con eventos tecnológicos pasados, o confiar en la red humana de apoyo que nos introduzca al uso tecnológico del artefacto.

Por último, el interlocutor se dispone a "manejar" el dispositivo y experimentar la interacción humana a través de las propiedades particulares que le ofrece la tecnología a su alcance. El interlocutor podrá superar, o restringirse a, un determinado espectro de posibilidades socio técnicas que estén a su alcance. Aunque podría pensarse que la frecuencia de la interacción está determinada por el artefacto, la realidad es que se encontrará ubicada en la calidad y alcance de las interacciones a través diversos paisajes tecnológicos y, dependerá del interlocutor y de su capacidad de integrarse a una sociedad, gestionar recursos e interactuar, en donde el conocimiento es la moneda de cambio.

Sirva, entonces, la figura 1 para sintetizar el proceso que los interlocutores tienen ante la tecnología. En ocasiones los procesos de relación con la tecnología obligan al uso del sentido común; sin embargo, podemos considerarlo como la síntesis del conocimiento que la humanidad, a través de los años, ha realizado para la comprensión de la tecnología en el desarrollo de sus sociedades e historias y tiene como objetivo hacer este resumen 
histórico, pero también situar al lector en alguna de las posiciones que puede tener frente a los procesos tecnológicos:

Figura 1: Esquema de los enfoques sobre la tecnología

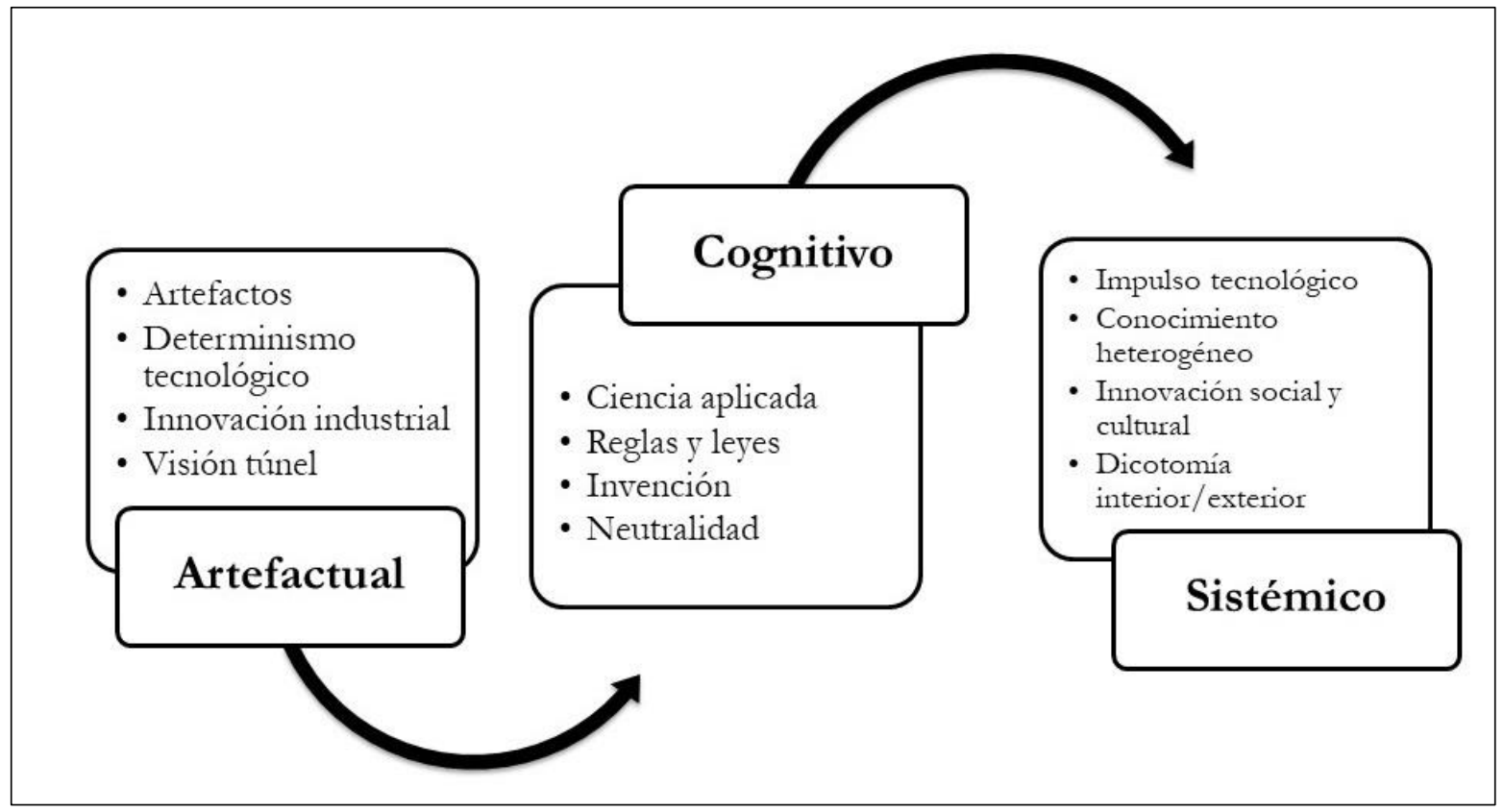

Fuente: Creación propia a partir de los textos de Heilbroner (1996), Bunge (1972), Hughes (1983)

\section{Estudios sociales de la ciencia}

Es imprescindible aclarar que los estudios acerca de tópicos como tecnología, sociedad, individuo y experiencia en la red de internet desde las Ciencias Sociales, se han desarrollado ya en la sociología, la antropología, la psicología social, la economía, la geografía, las ciencias políticas, los estudios de comunicación, estudios organizacionales y la sociolingüística, que incluyen estudios sobre temas de relaciones sociales en la red de internet. Entre los más destacados, están autores como Christiakis y Fowler (2010) los cuales sostienen un completo tratado al respecto, al analizar la influencia de la "red social" durante más de un siglo para denotar conjuntos complejos de relaciones entre miembros de los sistemas sociales en todas las dimensiones, desde el ámbito interpersonal hasta el internacional. En su libro Conectados (2010) debaten la influencia que ejercen sobre nosotros las amistades, los familiares y, en general, todo el mundo que conocemos. Y van más allá, incluyendo la influencia que ejercen sobre nosotros los amigos de nuestros amigos (aunque no los conozcamos) y hasta los amigos de los amigos de nuestros amigos en "... el sorprendente poder de las redes sociales y cómo nos afectan, que acaso parece un gancho para jóvenes geeks, este libro no habla de Twitter, Facebook o MySpace" (CHRISTIAKIS; FOWLER, 2010, p. 14). 
Seleccionar estas rutas es consolidar un esfuerzo teórico al intentar reunir reflexiones de carácter filosófico, histórico, sociológico, ético y político, complementándolas con referencias de los trabajos abordados desde ciencias sociales. Pretendo que esta síntesis interdisciplinaria nos dé como resultado una introducción a la comprensión de las interrelaciones entre ciencia, tecnología y sociedad. Las elaboraciones teóricas y las informaciones empíricas resultantes de esas aproximaciones pretenden, para esta investigación, aportar al conocimiento de las ciencias sociales en campos tan diversos como la educación científica, las políticas públicas en ciencia, tecnología e innovación y, desde luego, nuestro principal itinerario de investigación, la propia investigación académica sobre estudios en la red de internet y la comprensión de los fenómenos de interacción humana que ocurren en ella.

Desde la investigación antropológica y de estudios culturales estuvo se han encaminado esfuerzos para apreciar, documentar y analizar diversos procesos tecnológicos sobre internet (MENA, 2005). Destaqué temas tales como: a) la innovación tecnológica, b) los usos sociales de la tecnología, c) los entornos y cambios socio técnicos, d) los lenguajes tecnológicos y e) la construcción social de la tecnología (imágenes, representaciones, creencias en torno a los procesos y artefactos tecnológicos).

Lo anterior permite producir datos suficientes e interpretarlos mediante la comprensión del fenómeno tecnológico, situándome en un ejercicio que puede considerar transdisciplinar. La necesidad de abordar otros campos teóricos surge a partir del conocimiento empírico de nuevos saberes, prácticas y experiencias que me permitan indagar una parte acotada del conocimiento que se genera sobre la red de internet caracterizada como una tecnología considerada como emergente, pero al mismo tiempo hegemónica. Este conocimiento lo pretendo articular con un nuevo mapa tecnológico del mundo, muy distinto al que se conformó a lo largo del siglo XIX y primera mitad del siglo XX. Y es que basta con explorar empíricamente los procesos de recepción de las innovaciones, el consumo y las modificaciones de la vida cotidiana como un hecho académico altamente relevante, el cual se experimenta ante la presencia de las novedades tecnológicas en los años recientes y la dinámica de usos sociales. ${ }^{3}$

Si se contrasta el conocimiento empírico de uno o muchos individuos con las tradiciones teóricas del último medio siglo, se ha de reconocer ya a la tecnología como

\footnotetext{
${ }^{3}$ A principios del siglo XXI el consumidor de las nuevas tecnologías acepta, más y mejor, la evaluación de su desempeño, la productividad y la simple comunicación que suscita el uso de diversas tecnologías en su vida cotidiana y en las sociedades contemporáneas en las que es partícipe.
} 
un campo de estudio, y entonces podremos ejercer el derecho de analizar y comparar los criterios con los cuales los interlocutores definen su experiencia en ambientes tecnológicos diversos.

Los paradigmas más destacados, que no los únicos, desde donde las ciencias sociales han estudiado a las tecnologías, para después señalar cuál ha sido el sentido de reflexividad que constituye la más reciente fase del desarrollo de la nueva sociología del conocimiento científico y, por último, perfilar rutas teóricas metodológicas en torno a los estudios sociales sobre tecnología que aborda esta investigación.

En los programas de estudios de la ciencia se organizaron por primera vez como campo autónomo de los años cincuenta en diferentes contextos, continentes y escuelas teóricas, tratando de dar respuesta a demandas de pertinencia de la ciencia y tecnología ante corrientes políticas coyunturales. Un ejemplo de esto es el trabajo realizado por Merton (1953) en su libro Sociología del conocimiento, definió el tema de estudio de la sociología en tres puntos:
a) La génesis socio histórica de la ciencia moderna;
b) la ciencia como institución social y la constitución de un ethos específico en estas instituciones;
c) el problema de la estratificación social en las comunidades científicas y;
d) los problemas asociados a la generación que entre 1950 y 1960 experimentó un crecimiento tecnológico eléctrico y electrónico (MERTON, 1953, p. 35).

También dedicaron su esfuerzo a fortalecer estudios sobre la construcción de indicadores científicos; análisis del cambio en las políticas científicas gubernamentales; investigaciones sobre cultura y comunicación científica; estudios de las implicaciones sociales y medioambientales de la ciencia; valores amenazados por los cambios tecnológicos; estudios prácticos de controversias; investigaciones de actitudes y análisis de riesgos.

\section{La ciencia en acción en contra del determinismo}

En los ochentas, los enfoques anteriormente expuestos fueron aplicados al estudio de la tecnología a través de autores como Bijker (1987), Hughes (1983) y Pinch y Bijker (1987). ${ }^{4}$ La perspectiva analítica clásica modificó su rumbo y, en lugar de encontrar explicaciones en los fenómenos macro sociales, se enfocó en los fenómenos micro

\footnotetext{
${ }^{4}$ De acuerdo con Bijker (1987), Hughes (1983) y Pinch y Bijker (1987), investigadores en el área de la sociología de la tecnología, para los cuales la tecnología es construida socialmente. Los grupos sociales dan sentido a ésta, teniendo un papel fundamental en la significación y solución de los dilemas que se forman en la trayectoria y uso de los artefactos tecnológicos, dotando de una óptica de uso y posibilidades a éstos, que pueden ser diferentes de los objetivos institucionales que se les han asignado.
} 
sociales. Así, por ejemplo, los llamados "estudios de laboratorio" reducen el contexto social al contexto del laboratorio e intentan así comprender la "ciencia en el proceso de ser hecha".

Emergieron varios campos afines para explorar determinadas implicaciones de la ciencia y la tecnología: estudios de bioética y ética en las ciencias médicas, y consecuencias de los cambios tecnológicos en el medio ambiente. En los ochentas, los principales debates fueron sobre el determinismo tecnológico y sobre la construcción social de la ciencia y la tecnología. En ellos se desplazó el interés del impacto social de la ciencia y la tecnología a los procesos sociales de la investigación y las maneras en que la ciencia es moldeada y permeada por los valores sociales.

Los estudios sobre la estructura social interna de la ciencia prevalecientes en la tradición mertoniana (del 1940 al 1970) comienzan a ser desplazados por los estudios de la “ciencia en acción” de Latour (2008), orientados a explorar los procesos que desarrolla el conocimiento científico, cómo son creados o construidos, incorporados y desestimados los hechos científicos y cómo ocurren los procesos de legitimación y de descalificación como pseudociencia.

En estos años la etnografía fue considerada como la estrategia para crear datos y establecer líneas de análisis, paso trascendental para que en los años noventa se empezara a ver a la ciencia como cultura, en donde por primera vez la ciencia ya no se acepta como algo ajeno a los códigos culturales, a las fuerzas sociales y económicas, y a los intereses profesionales. Estos análisis, que causan gran consternación entre muchos científicos, tratan a la ciencia como un producto social y cultural, y a la comunidad científica como mano de obra. Fundamentalmente, los estudios culturales se han orientado a la interpretación de las narrativas y discursos científicos y sus expresiones en los circuitos mediáticos, desde la prensa hasta el internet.

\section{La etnometodología de la actividad científica}

Uno de los aportes más significativos de las ciencias sociales a los estudios de los fenómenos científicos y tecnológicos se da con el uso de la etnometodología. Su aplicación en los estudios de la actividad científica se debió a que ésta aporta una larga tradición de trabajo y análisis de las actividades y circunstancias prácticas y en cómo estas últimas constituyen, en el curso de las continuas interacciones localmente situadas, la vida cotidiana de los individuos y, por consecuencia, conforman el orden social. 
Sin embargo, esta orientación metodológica no pretende abordar las realidades humanas o sociales desde cero, sino que usa, con prudencia, los recursos que la sociedad en cuestión le ofrece. Así, el trabajo de interpretación estará influido por instrumentos interpretativos locales, como categorías reconocidas, vocabulario familiar, tareas organizativas, orientaciones profesionales, cultura grupal y otros marcos conceptuales que les asignan significado a los asuntos a considerar.

Fundamentalmente, la corriente etnometodología se ha aplicado a las labores que desarrollan los científicos en sus lugares habituales de trabajo, y se ocupa de caracterizar las prácticas diarias de la actividad científica y tecnológica, buscando conocer la manera en que los diversos actores identificados en estos contextos construyen y mantienen sus órdenes sociales de referencia. Se toma en cuenta el análisis conversacional (tema favorito de la tradición etnometodológica) propuestos por Garfinkel (2006) y sus discípulos Lynch (2000) y Livingston. Ellos referencian las prácticas que permitan distinguir actividades científicas de las no científicas, y la propuesta del planteamiento etnometodológico sostiene que la vida científica es una actividad cotidiana y ordinaria más, organizada de modo natural, y que es posible identificarla por una serie de rasgos idénticos a otro tipo de prácticas sociales.

Los estudios etnometodológicos de la ciencia se dirigen al análisis empírico de las relaciones que se establecen entre la acción (sistemas de competencia autóctono), como son las conversaciones de los actores vertidas en textos escritos, las pruebas matemáticas, los dibujos, las fotografías, en fin, todo aquello que identifique los aspectos cotidianos, que terminan por tornarse rutinarios y que nos permiten describir cada supuesto frente a las pretendidas instrucciones universales que el método científico proporciona.

La etnometodología nos permite observar acciones y prácticas de acuerdo con criterios establecidos tales como que sea posible: "[...] localizar un dominio indefinidamente amplio de escenarios apropiados [...] en donde encontramos el sentido, factibilidad, objetividad, causa, explicación y comunidad de las acciones prácticas" (GARFINKEL, 2006, p. 42).

Lo anterior posibilita que las actividades cotidianas de los interlocutores dentro de los paisajes tecnológicos proporcionen contextos, objetos, recursos, justificaciones, tópicos, problemáticas y, en resumen, prácticas y productos de investigación de nuestro interés. Además, permite que los escenarios de la investigación se puedan observar cómo: “...los miembros de un arreglo organizado estén constantemente obligados a decidir, 
reconocer, persuadir o hacer evidente el carácter racional (coherente) de sus acciones" (GARFINKEL, 2006, p 43).

Y por tanto, existe una referencia a que los fenómenos son prácticas comunes de la organización y están variablemente disponibles y, en el caso de las experiencias en la red, se identificarán a partir de la producción discursiva.

Nos permite encontrar la evidencia de un espacio y de ciertas acciones que estamos en posibilidad de atestiguar “...la reproducción de las actividades — es decir; que las propiedades racionales de actividades prácticas-, las cuales son tomadas en cuenta, reconocidas, categorizadas, descritas, usadas, producidas y tratadas por los miembros del escenario" (GARFINKEL, 2006, p 44).

Con ello evidenciar la posibilidad de entender que los procedimientos de reproducción de actividades que tienen propiedades lógicas y metodológicas de las prácticas, y que sus características generales son de interés para el estudio fenomenológico. Las actividades prácticas organizadas de la vida cotidiana que difieren estructuralmente. Deben ser buscadas y analizadas en cuanto a su producción, orígenes, reconocimiento y representaciones de prácticas racionales.

Para la etnometodología es recomendable que el criterio de análisis en cualquier escenario social “... sea visto como auto-organizador, con respecto al carácter inteligible de sus propias manifestaciones como representaciones o evidencias del orden social" (GARFINKEL, 2006, p 44). O sea que cualquiera de las experiencias de la vida cotidiana tiene el carácter de actividad práctica, contable, detectable, narrable, analizable, en resumen, explicable y por tanto:

Toda forma de investigación, sin excepción, consiste en ingeniosas prácticas organizadas por las cuales se vuelven evidentes o se demuestran propiedades [...] de las expresiones y acciones contextuales, las cuales son un logro de las actividades de la vida cotidiana (GARFINKEL, 2006, p. 45).

De ahí el carácter importante de la etnometodología, ya que propone que la producción gestionada del fenómeno, en todos sus aspectos, conserva el carácter de tarea práctica, seria y sujeta a cada exigencia de la conducta organizacionalmente situada.

Por lo tanto, la etnometodología nos permite encontrar el sentido a las acciones prácticas, reconocer el carácter racional (coherente) de las acciones de los sujetos, situar acciones contextuales en la vida cotidiana, observar al escenario social como autoorganizador $\mathrm{y}$, por último, evidenciar las propiedades de las expresiones y acciones contextuales de interlocutores en red. 
En concreto, la etnometodología apoya a sistematizar los hallazgos en el trabajo de campo, tales como la sistematización de conversaciones que permitan identificar por lo menos cuatro variables que estructuran al texto y faculten su lectura específica. Éstas son:

a) la instrucción preliminar que nos permita conocer la afiliación institucional del autor, algunas características relevantes del texto, y asignar el sentido concreto de distintos paisajes;

b) los mecanismos de externalización, que proporcionan la imagen del hecho considerándolo que tiene existencia por sí mismo, y ubicándolo más allá de las subjetividades humanas.

b) los mecanismos de conducción, que permiten fijar o establecer estados de cosas pasadas y dan cuenta de cómo han podido capturarse los objetos identificados, impidiendo que se cuestione cualquier dato o aspecto que aparezca en el texto;

c) los mecanismos de secuenciación, que permiten ordenar los acontecimientos en la narración, desechando otros caminos potenciales y destacando acontecimientos significativos según Woolgar (ESPINOSA; GINER; TORRES, 2002, p. 557-571).

\section{La reflexividad en la organización social}

Entre los estudios más recientes sobre la sociología del conocimiento científico, tenemos los relacionados con la representación o exploración de la reflexividad en la organización social de la ciencia y la tecnología. Este tipo de estudios los encabezan Kreimer y Hernán (2004), Beck (1998), Giddens (1999), Lash (2005), Winner (1993) Woolgar (1991) y Hughes (1983).

La propuesta de Kreimer y Hernán (2004) es sobre los marcos analíticos y las metodologías de investigación empleadas para "mirar sobre las condiciones periféricas bajo las que se produce, se negocia y se usa el conocimiento científico y tecnológico" (KREIMER; HERNÁN, 2004, p. 77) y, con ello, la generación de agendas de investigación y acción, las cuales comprenden sus compromisos con la región en la cual se implementan dichas tecnologías.

Estos estudios proponen diversas líneas de análisis y definen tres ámbitos de la investigación sociológica sobre la ciencia y tecnología como lo son: la construcción social de la tecnología; la teoría del actor-red y el modelo de los sistemas

De ellas se desprende un vasto temario de análisis para la investigación sobre temas de tecnología. A continuación, enlisto los más relevantes:

a) La relación que guarda la organización social de la ciencia y la tecnología con las estructuras organizacionales;

b) el proceso de estratificación social en las comunidades científicas y tecnológicas; 
c) el estudio sobre trayectorias formativas de científicos y tecnólogos, y sus nexos con las trayectorias disciplinarias, sub disciplinarias y campos emergentes;

d) el estudio sobre procesos de tecnología alternativa;

e) el estudio sobre constitución de "grupos de presión" contestatarios de tecnólogos, científicos y ciudadanos (ambientalistas, ecólogos, grupos religiosos, sindicatos, hackers) contra las tecnologías de riesgo;

f) el estudio de innovación científica y tecnológica que se concentra en los procesos de invención científica y de artefactos técnicos;

g) el estudio de recepción de la tecnología a partir de una sociología del consumo y una teoría de las representaciones sociales (procedente de la psicología social) para comprender los usos sociales de la tecnología y los procesos de construcción de los significados tecnológicos;

h) el estudio sobre los impactos tecnológicos y cambio socio técnico, los cuales tratan de documentar cómo las novedades técnicas modifican los entornos sociales y cómo se articulan procesos de resistencia, asimilación o reconversión social de tales impactos de artefactos o ambientes tecnológicos;

i) el estudio sobre historia social de la tecnología, que contribuye significativamente a documentar los procesos tecnológicos desde una perspectiva histórica, proveyendo al resto de los enfoques de argumentos para sus análisis, constituye una perspectiva metodológica en sí misma;

j) el estudio económico sobre los procesos tecnológicos, especialmente se centran en los nexos empresa, empresario, tecnólogos, invención, mercados, precios, comercialización y competencia; y

k) la exploración de los usos reales, fácticos, de las nuevas tecnologías.

De todos ellos, esta investigación se identifica con los temas referentes a la Construcción Social de la Tecnología, la cual intenta dar respuesta a lo que denuncian como las "visiones lineales y deterministas" de la mayoría de los estudios en Historia y Sociología de la Tecnología, un grupo de intelectuales norteamericanos y europeos ha venido trabajando, desde finales de los años sesenta, en la consolidación de un nuevo conjunto de herramientas teóricas que permitan repensar la historia sobre la tecnología, usando como ejemplo el uso social de artefactos. Un ejemplo es la ya clásica reevaluación de la historia de la bicicleta aplicando nuevas herramientas metodológicas. Pinch y Bijker 
(1987), nos muestran cómo la historia popular y heroica de la bicicleta se desarrolla en Estados Unidos y se resume en una evolución del artefacto que poco a poco va pasando de diseños pobres e inapropiados a uno que finalmente se consolida como el mejor gracias a sus ventajas técnicas.

Hombres y mujeres experimentan en la actualidad historias, experiencias y adaptaciones muy similares en el uso de las primeras computadoras, las primeras redes de internet y hasta las redes sociales de hoy en día. El ejemplo de la construcción social puede ser usado entonces para reconstruir y explicar las trayectorias particulares del desarrollo del artefacto, introduciendo la noción de flexibilidad interpretativa (BIJKER, 1987). Es decir, los significados radicalmente diferentes del artefacto pueden ser identificados por los distintos grupos sociales involucrados. La flexibilidad interpretativa puede llegar a cancelarse, es decir, a prevalecer uno de los significados en escena, dado el proceso o mecanismo de cierre o de clausura.

Estamos en condiciones de usar la noción de marco tecnológico, refiriéndose a un marco de significado relacionado con una tecnología en particular, en el caso de esta investigación, hablamos de la tecnología internet compartida entre varios grupos sociales en todo el mundo y que además guía y da forma al desarrollo de los artefactos (PINCH; BIJKER, 1997, p. 26-28). En resumen, esta investigación se apoya en:

a) en la construcción social de los significados tecnológicos;

b) dar lugar preponderante a las trayectorias (o biografías) sociales de los artefactos tecnológicos

c) en los procesos interpretativos de resignificación y sus tensiones inherentes para hacer prevalecer o cohabitar un punto de vista sobre los artefactos técnicos;

d) la dimensión de la representación social y las percepciones sociales como cruciales para el análisis social de la tecnología.

Lo anterior nos permite situar a la investigación en la Teoría del actor-red, que si bien parte del enfoque construccionista es menos radical en cuanto le da mayor cabida a "la dimensión material de la tecnología, al incluir la interacción entre actores humanos y no humanos" (LAW, 1992, p. 385). El actor-red se distingue del actor tradicional de la sociología, cuya categoría excluye generalmente cualquier componente no humano. No se reduce ni a un simple actor ni a una red. Está compuesto, al igual que las redes, de series de elementos heterogéneos, animados o inanimados, que han sido ligados mutuamente durante un cierto periodo de tiempo. Un actor-red es, simultáneamente, un 
actor cuya actividad consiste en entrelazar elementos heterogéneos y una red que es capaz de redefinir y transformar aquello de lo que está hecho. La relación entre ciencias sociales y tecnología es explicada por este modelo a partir de la siguiente afirmación de Callon (1998):

Transformar la sociología académica en una sociología capaz de seguir a la tecnología a lo largo de su elaboración significa reconocer que el objeto de estudio apropiado no es ni la sociedad misma ni las así llamadas relaciones sociales, sino los actores-red que dan lugar, simultáneamente, a la sociedad y a la tecnología (CALLON, 1998, p. 163).

Por último, un modelo que puede apoyar el análisis de los datos de cualquier investigación en ambientes tecnológicos podría apoyarse en el Modelo de Sistemas, que fue introducido por Hughes (1983) como estudio de los sistemas socio técnico. Éste plantea que el proceso tecnológico se compone de varias fases: invención, desarrollo, innovación, transferencia, crecimiento, competición, y consolidación. Desde este modelo las tecnologías pueden ser analizadas las relaciones socio técnicas como sistemas con componentes heterogéneos; se constituyen en un modelo sistémico que incluye procesos tales como: concepción-invención/ experimentación-producción/ persuasión, distribución consumo, retroalimentación, reconcepción, ajustes, internalización cotidiana. Lo anterior implica incorporar al análisis sistémico las diferentes instancias que intervienen en dichos procesos: empresas, estados, consumidores, tecnólogos, publicitarios. Supone observar las diversas dimensiones en las que el fenómeno ofrece su estructuración sistémica: ecología cultural, aspectos políticos, legislativos, culturales, económicos, discursivos, iconográficos, financieros, ergonómicos, espaciotemporales, organizativos, científicos. Sin embargo, este modelo privilegia los enfoques macro en la articulación de los análisis, aunque no descarta su utilización en modelos micro.

A manera de resumen y con el fin de poner en marcha lo anteriormente expuesto, se presenta la figura 2, que sintetiza los modelos teóricos y las categorías de análisis que se proponen para llevar acabo cualquier investigación. Esto tiene como objetivo conectar algunos de los posicionamientos teóricos con el trabajo empírico y analítico y poder perfilar las categorías de análisis que permitirán a quienes investiguen la red de internet, realizar análisis en el proceso de la producción de datos en el trabajo de campo. 
Figura 2: Esquema de los Modelos teóricos y categorías analíticas.

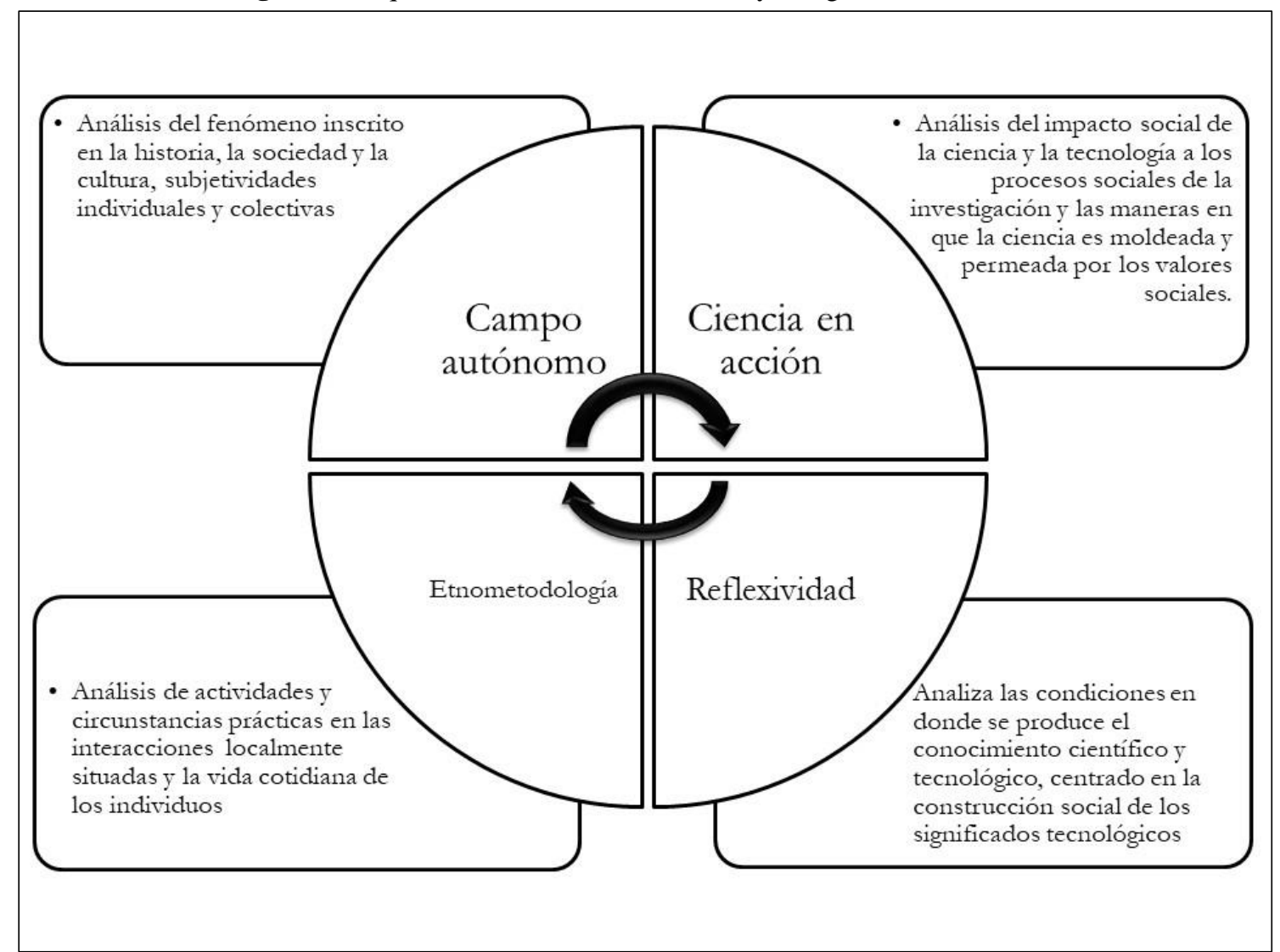

Fuente: Creación propia a partir de Nelkin (1998), Garfinkel (2006), Espinosa, Giner y Torres., (2002), Kreimer y Hernán (2004).

De igual forma que en los esquemas anteriores, la figura 3 se presentan, a manera de resumen, los tres modelos de análisis antes descritos. Su visualización de forma sintética nos permite caracterizar las relaciones con la tecnología, el actor y, por supuesto, el modelo de sistema, y representa tres vertientes de análisis en los fenómenos dentro de la red de internet. 
Figura 3: Modelos de análisis de estudios sociales sobre tecnología

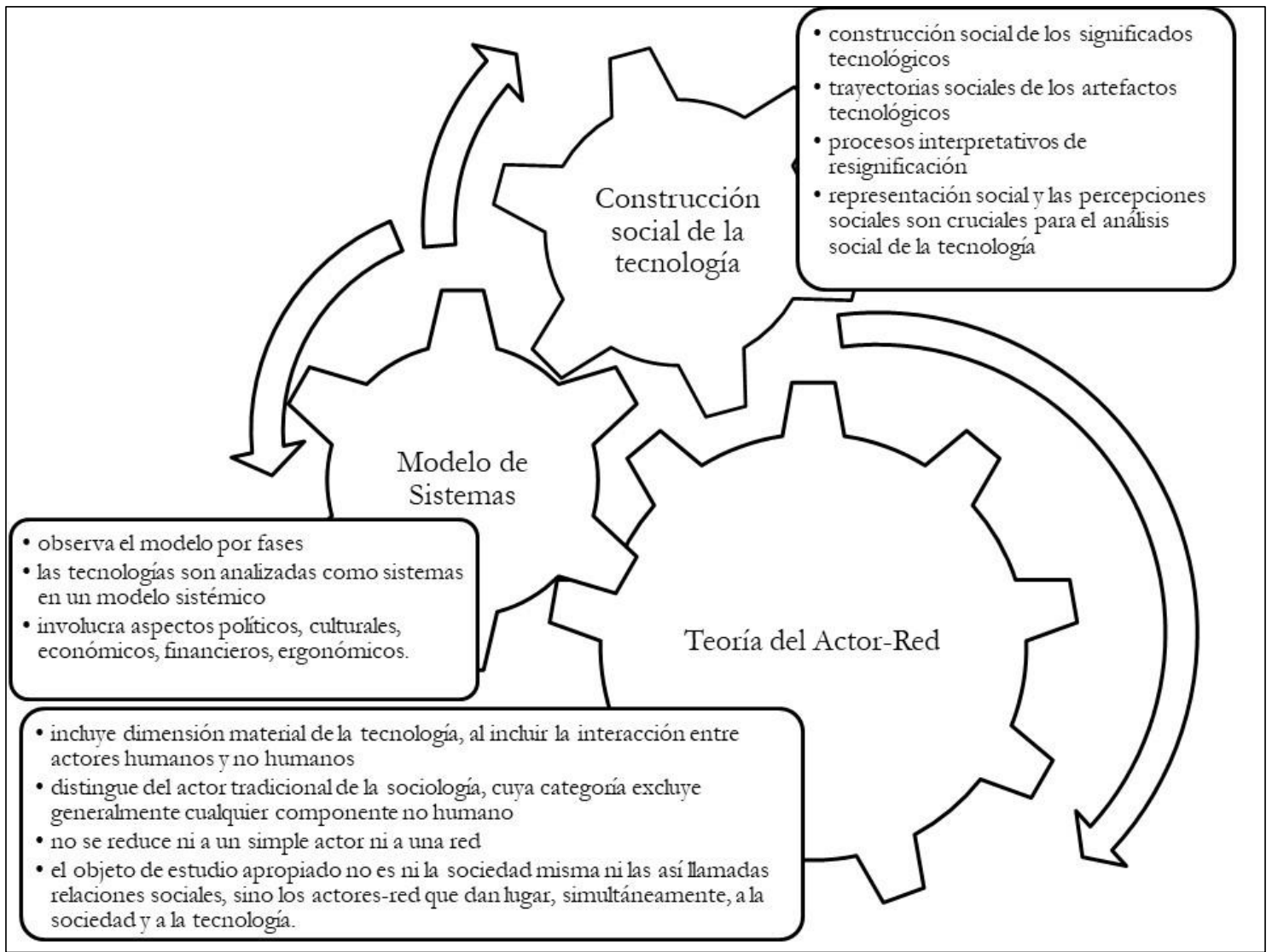

Fuente: Creación propia a partir de Winner (1993), Bijker, (1987), Pinch y Bijker (1987), Hughes (1983), Law (1992) y Callon (1998).

\section{Internet, un reto teórico en la fenomenología tecnológica}

Si bien, las anteriores perspectivas teóricas orientan el análisis de los efectos que tienen la tecnología, la ciencia y la comunicación, en diferentes ámbitos y momentos del desarrollo tecnológico de la humanidad, un reto pendiente es el iniciar un camino hacia la comprensión teórica que abstraiga los fenómenos que produce la interacción de millones de seres humanos a través de la red de internet. Por ello un posible análisis se apoya en un posicionamiento fenomenológico y etnometodológico debido a que las perspectivas teóricas de los apartados anteriores nos orientan a un reducido aspecto del análisis de la experiencia tecnológicas. Los estudios en internet aspiran sin duda a la comprensión de los efectos que tienen la tecnología, la ciencia y la comunicación, en diferentes ámbitos y momentos del desarrollo tecnológico de la humanidad. Lash (2005) lo desarrolla con la fenomenología tecnológica, en su libro Crítica de la Información en donde analiza los principios estructurales de la Cultura Global de la Información respecto de la sociedad industrial y estudia los bienes de consumo (objetos) mientras se mueven con velocidad a través de las redes globales. A diferencia de las antiguas estructuras 
sociales del orden industrial (sujeto protagonista), el nuevo orden mundial de la información favorece la aparición de objetos autónomos. Los objetos o artefactos son autónomos; es decir, escapan al control de los sujetos a través de las redes globales constituyéndose en flujos (signos, medios, migrantes, tecnología, dinero). De esta manera, los objetos pasan a compartir cualidades informacionales (la forma de información y comunicación).

Lash (2005) sitúa el cambio de una cultura representacional a una cultura tecnológica, en donde el dualismo sujeto-objeto, es remplazado por una dimensión inmanente a la sociedad la cual no la trasciende ni está por encima de ella y, por lo tanto, requiere de la reflexividad la cual se refiere al vínculo entre el conocimiento y la acción, planteada por Garfinkel (2006) el cual se inclina por una fenomenología radical de las comunicaciones en la que lo intangible y lo material se "comprimen" en una única unidad comunicacional, y se opone a las primeras fenomenologías trascendentales como la defendida por Husserl (1997).

Lo anterior implica que pasemos del observador, que puede prestar o no atención a las cosas, produce conocimiento del objeto por medio de juicios y la duda, y conoce en términos de causa y efectos, a un experimentador, que conoce gracias a una actitud en la perspectiva de su intencionalidad, en menos causal que descriptivo, que no explica sino interpreta y que basa su conocimiento a través de la experiencia.

Tanto Lash (2005) como Garfinkel (2006) proponen un camino que va de la actitud natural (el plano empírico), pasando por una actitud científica (una forma de ver al mundo que se observa desde otro mundo, manifestándose como atemporal e inextensa) a una actitud reflexiva, que propone que la descripción no produce un conocimiento a priori (kantiano) sino que es demostrativo, convincente, ósea apodíctico, que es el conocimiento de las cosas en sí.

Lash (2005) propone que la etnometodología que resuelva el qué pero sobretodo del cómo. Responder el qué es la interpretación y descripción fenomenológica de cosas y acontecimientos a partir de ámbitos que son las actitudes. En tanto responder el cómo es en sí un modo de alcanzar el conocimiento, realizada por descripciones empíricas, en donde el conocimiento es socialmente organizado y práctico.

Así que hablar de una fenomenología empírica de las comunicaciones, según Garfinkel es "responde a qué es lo reflexivo, siendo esto las "prácticas descriptivas" dadas en la colectividad, en donde se le da congruencia a la realidad, y en donde "el contar" da su carácter central de las comunicaciones” (2006, p. 65). El cumplimiento de las prácticas 
descriptivas (comentarios) aseguran la reproducción de las actividades Por tanto los comentarios en las conversaciones y el cómo de las mismas son consideradas como actividades extendidas en el tiempo, en donde no solo están "ahí" sino constantemente "ahora".

Un cambio fundamental con la vieja fenomenología es que para Lash (2005) y Garfinkel (2006) no hay esencias, por tanto, no hay cosas en si para conocer o no conocer, sino que solo las cosas, tal como se manifiestan los actores interesados en una supuesta inmanencia. La comunicación de formas particulares y no son descripciones de las esencias de las cosas, sino son glosas o comentarios de actividades y expresiones. Entonces, la unidad de análisis para Garfinkel (2006) son esas relaciones extendidas de intersubjetividad, son ordenamientos sociales, los cuales se dan por medio de una cultura tecnológica en donde el tiempo de interrupción no existe debido a las comunicaciones a distancia por intermediación de las máquinas

Para poder operar dentro de una fenomenología tecnológica conceptualizaremos el lugar en donde el estudio se realizó. Para ello se presentan seis apartados, los cuales tienen como intención caracterizar al fenómeno, e iniciar una conceptualización de términos que nos lleven a la construcción de categorías analíticas de análisis del fenómeno de interacción en la red.

\section{Conclusiones}

El articulo presenta las distintas posibilidades metodológicas de análisis y comprensión de las prácticas y significados de las experiencias humanas que son escritas, publicadas, difundidas, compartidas e interpeladas en la red de internet, las cuales estimulan un sinnúmero de relaciones socio técnicas ${ }^{5}$ que refieren a un entramado complejo de relaciones y asociaciones formadas por múltiples y heterogéneas entidades.

Inicia con las formas en que han sido abordados los temas acerca de tecnología y concluye con la propuesta metodológica que ofrece Lash (2005) sobre la fenomenología tecnológica y los de Garfinkel (2006) sobre la etnometodología. La primera permite “...explicar que en la cultura tecnológica, el conocimiento ya no está por encima de la

\footnotetext{
${ }^{5}$ Habitualmente, cuando se habla de relaciones sociotécnicas se suele mencionar que este entramado actúa como dispositivo. Significa que su acción genera unos efectos que se pueden prever a partir de la conexión de todos sus elementos, así como de otros que son completamente inesperados e imposibles de predecir. Las relaciones en las redes sociotécnicas muestran que vivimos en un mundo sostenido "por broches" tecnológicos en el cual es imposible decir dónde inician y terminan la relación sociotécnica y la sociocultural.
} 
naturaleza social [...] ni la trasciende, sino está inmanente a ella" (LASH, 2005, p. 264). En la fenomenología tecnológica existe una reflexividad que ya no tiene que ver con la toma de decisiones distanciadas ni con la organización de relatos de vida por sí mismos, sino se refiere al cambio en el vínculo reflexivo del conocimiento y la acción, de modo que ya no existe ninguna distancia entre ambos. La acción es conocimiento y viceversa.

Esta relación sencilla pero poderosa rompe con el humanismo efectivo y los supuestos ontológicos de los primeros fenomenólogos que se sitúan en la escuela de pensamiento filosófico clásico — trascendental y del mundo de la vida—, defendida por Husserl (1997) y que, desde su inicio, propone el rompimiento con la lógica de la representación al suponer que:

[...] el sujeto deja de estar por encima del mundo de los objetos y las relaciones sociales. No lo suponía por encima del mundo, sino en el mundo con los objetos y las relaciones sociales. Y no lo consideraba neutral sino poseedor de una intencionalidad, una actitud hacia el mundo (LASH, 2005, p. 210.).

En tanto que para Garfinkel (2006) el cómo y el qué son inseparables y llevan a poner la atención en el sujeto, el actor o en nuestro caso el interlocutor en la red de internet. Lo que produzcamos en la red y será en interacción con los lectores o escritores de textos, y por tanto retoman una actitud que: “[...] maximiza su presencia en el mundo, el carácter de nuestras actitudes hacia las cosas, las expresiones y los procesos sociales en el mundo" (GARFINKEL, 2006, p. 286).

\section{Referencias}

BECK, U. La sociedad del riesgo. Hacia una nueva modernidad. 1. ed. Barcelona: Paidós. 1998.

BIJKER, W. La construcción social de la baquelita. Hacia una teoría de la invención. Ciencia, Tecnología y Sociedad, Lecturas seleccionadas, Barcelona, p. 103-129. 1987.

BUNGE, M. La Investigación Científica, Su estrategia y su Filosofía. 1 ed. Ediciones Ariel: Barcelona, 1972.

CALLON, M. El proceso de construcción de la sociedad. El estudio de la tecnología como herramienta para el análisis sociológico. Sociología Simétrica, [S.I.], p. 143-170. 1998.

CHRISTAKIS, N.; FOWLER, J. Conectados. 1 ed. Taurus: Madrid, 2010.

ELLUL, J. El siglo XX y la técnica: análisis de las conquistas y peligros de la técnica de nuestro tiempo. 1 ed. Barcelona: Editorial Labor, 1960.

ESPINOSA, L.; GINER, S.; TORRES, C. Diccionario de Sociología. 2 ed. Alianza Editorial: Madrid, 2002. 
GARFINKEL, H. Estudios en Etnometodología. 1. ed. Anthropos: Barcelona. 2006.

GIDDENS, A. Modernidad y autoidentidad. Las consecuencias perversas de la modernidad. 1. ed. Anthropos: Barcelona. 1999.

HABERMAS, J. Teoría de la acción comunicativa. 1. ed. Paidós: Barcelona. 1988.

HEILBRONER, R. ¿Son las máquinas el motor de la historia? 1. ed. Tecnos: Madrid. 1996.

HUGHES, T. Networks of Power: Electrification in western society, 1880-1930. Baltimore: Johns Hopkins University Press, 1983.

HUSSERL, E. Ideas relativas a una fenomenología pura y una filosofía fenomenológica. 1. ed. Traducción de José Gaos. FCE: México. 1997.

KREIMER, P.; HERNÁN. T. Un poco de reflexividad o ¿de dónde venimos? Estudios sociales de la ciencia y la tecnología en América Latina. En: KREIMER, P. et al. (eds.). Producción y uso social de conocimientos, Estudios de sociología de la ciencia en América Latina. 1. ed. Bernal: Universidad Nacional de Quilmes, 2004. p. 11-90.

LASH, S. Critica de la información. 2. ed. Buenos Aires: Amorortu, 2005.

LATOUR, B. Reensamblar lo social: una introducción a la teoría del actor-red. 1. ed. Buenos Aires: Ediciones Manantial, 2008.

LAW, J. Shaping technology, building society: studies in sociotechnical change. 1. ed. Cambridge: MIT Press, 1992.

LYNCH, M. Against reflexivity as an academic virtue and source of privileged knowledge, Theory, Culture and Society, London, v. 17, n. 3, p. 27-53, 2000.

MENA, R. Cibercultura y ciberespacio en San Cristóbal de las Casas, Chiapas. La construcción de comunidades sociales y culturales interconectadas por las tecnologías de información en una ciudad del sureste de México. 2005. Tesis de licenciatura en Antropología Social - Facultadde Antropología Social, Universidad Autónoma de Chiapas, México, 2005.

MERTON, R. K. Sociología del conocimiento. 1. ed. Buenos Aires: Editorial Deucalion, 1953.

NELKIN, D. Tecnociencia y cibercultura.1 ed. Barceloba: Editorial Paidos. 1998.

PINCH, T.; BIJKER, W. The Social Construction of Facts and Artifacts: Or How the Sociology of Science and the Sociology of Technology Might Benefit Each Other. En: BIJKER, W.; HUGHES, T.; PINCH, T. (eds.). The Social Construttion of Tecbnological S)'stems. Neu. Directions in tbe Sociology and Hútor of Technology. 1. ed. USA: MIT Press, 1987. p. 15-50

QUINTANILLA, M. Tecnología: un enfoque filosófico. 2. ed. Madrid: Paidós. 2001.

SANTAMARINA, C.; MARINAS, J, M. Metodología de la Investigación III. Notas del curso en el Doctorado en Ciencias Sociales y Humanísticas, México, CESMECA-UNICACH. 2007.

SMITH, R.; MARX, L. Historia y Determinismo. Madrid: Editorial Alianza, 1996.

THOMAS, H. Tecnologias para Inclusão Social e Políticas Públicas na América. En; DJAMEL, N.; GUSTAVO, D.; BUZAI, A. (coord). Cibergeografia. Tecnologia de la informacion y 
lascomunciaciones en las nuevas visions espaciales. 1 ed. Mexico: Universidad Autonoma de Baja California. 2009. p. 41-78

WINNER, L. Social Constructivism. Opening the Black Box and Finding it Empty. USA: Saence as Culture. 1993.

WINNER, L. Tecnología autónoma. Barcelona: Gustavo Gilli. 1993 [1979].

WOOLGAR, S. Ciencia: abriendo la caja negra. 1. ed. Barcelona: Anthropos, 1991.

WYNNE, B. Redefining the Issues of Risk and Public Acceptance. Technology as a Social and Political Phenomenon. Nueva York: Academic Press, 1983.

Recebido em: 06 de setembro de 2018.

Aceito em: 20 de julho de 2019. 